\title{
Ab initio Modeling of Optical Properties of Organic Molecules and Molecular Complexes
}

\author{
Vladimir I. Gavrilenko \\ Center for Materials Research, Norfolk State University, 700 Park Avenue, Norfolk VA \\ 23504, USA \\ vgavrilenko@nsu.edu
}

\begin{abstract}
Electronic excitations are key points of most of the commonly measured optical spectra. The first principle studies of excited states however require much larger effort than computations of the ground state reliably reproduced by the density functional theory (DFT). In present work computation of optical functions of organic molecular complexes is studied. The system of independent particles excited by external light field is considered within perturbation theory (the random phase approximation, RPA). Optical response functions are calculated using $a b$ initio pseudopotentials theory. Results of predicted optical absorption associated with organic semi-conducting conjugated polymers, poly-phenylene-vinylenes (PPV), are presented. Effects of different corrections to the DFT improving accuracy are considered. Results are discussed in comparison with available experimental data.
\end{abstract}

\section{Introduction}

It has been demonstrated for decades that Kohn-Sham density functional theory (DFT) realistically predicts electronic structure of different systems, such as atoms, molecules, and solids [1 to 3]. Various generalized gradient approximation (GGA) methods describing exchange and correlation (XC) interaction have been shown to systematically improve the local density approximation (LDA) predictions of equilibrium atomic geometries [3]. In atomic systems (like large single molecules, inorganic solids etc.) the geometry optimization study primarily requires accurate prediction of the ground state which is well reproduced within a standard DFT approach. Electronic excitations are key points of most of the commonly measured optical spectra of organic materials (see [4] and references therein). The first principle studies of excited states however require much larger effort than computations of the ground state. The DFT eigen energies underestimate gaps between bonding and antibonding states which requires quasi-particle $(\mathrm{QP})$ correction [2,3]. It has been proved that in inorganic solids [5] and organic polymers [2] the QP correction results in substantial overestimates of the gaps. The situation is substantially improved by additional inclusion of excitonic (many-body) interaction into Hamiltonian of the system $[2,3]$. This state-of-the-art DFT+QP many-body theory predicts excitation energies in organic polymers with a good accuracy [2]. Such approach however 
requires large scale computations and it is very time consuming. On the other hand the corrections for QP and exciton interaction in electron eigen energies could be included through the scissors operator $[3,5]$.

In this work a first principle method to predict optical functions of complex organic molecules and molecular complexes based on ab initio pseudopotential (PP) theory is described. Corrections for QP shift and excitonic effects are incorporated through the scissor operator. This method could be used to study electronic structure and optical functions of complex molecules and complexes which is demonstrated for organic polymer calculations.

\section{Method}

Optical absorption spectra of organic conjugated poly-phenylene-vinylenes (PPV) polymers with linear decacyl $\left(\mathrm{C}_{10} \mathrm{H}_{21}\right)$ groups (donor RO-PPV block) are calculated using $a b$ initio pseudopotentials method. For this system an infinitely long PPV polymer chain is considered. This approach is distinctly different from that traditionally used in quantum-mechanical calculations [4]. Advantages of this approach were demonstrated for trans-polyacetylene and simple PPV polymers [2]. Recently we applied this method for optical absorption study of Rhodamine 6G (R6G) dye molecular aggregates [6].

Here both commercial [7] and research [8] computational packages based on $a b$ initio PP are used. In order to apply pseudopotentials in molecular system we use the super-cell method [9]. The electron energy structure and eigen-functions are calculated within DFT-GGA using fully separable $a b$ initio PP generated according to the Troullier-Martin scheme [10]. Energy cut-off up to $60 \mathrm{Ry}$ is taken to generate fully separable PP [8]. Convergence of the ground state has been proved by test calculations with energy cutoff $\left(E_{c u t}\right)$ up to 100 Ry for R6G single molecule.

Accuracy of better then $10 \%$ for eigen values is achieved with $E_{c u t}=45 \mathrm{Ry}$. This value is used in this work in order to avoid complexity. For atomic relaxation of single molecule and molecular dimers the super cell method is used. The PerdewBurke-Ernzerhof (PBE) functional was used to model XC interaction [11].

Optical absorption spectra are calculated within RPA approach [5, 8, 9] according to $\alpha(\omega)=2 \omega k(\omega)$ [12].The extinction coefficient $k$ is determined through real and imaginary parts of the dielectric permittivity function $\varepsilon(\omega)=\varepsilon_{1}(\omega)+i \varepsilon_{2}(\omega)$. The evaluation of $\varepsilon_{\alpha \beta}(\omega),(\alpha, \beta=x, y, z)$, in this work is based on the independent particle approximation. We consider the effect of gauge invariance for computation of $\varepsilon_{\alpha \beta}(\omega)$ (details are given in Appendix). The time dependent linear optical susceptibility function follows from the equation of motion for the density matrix (the RPA picture) [5,9]. The imaginary part of the function of $\varepsilon_{\alpha \beta}(\omega)$ in Coulomb (or velocity) gauge and in length gauge is given by Eq (16) or Eq (17) respectively. The real part is calculated using Kramers-Kronig formula [12]. 


\section{Results and Discussion}

In order to study the effect of the gauge invariance on predicted optical spectra of organic molecules the optical absorption spectra of R6G molecular dimmers [6] and of RO-PPV polymer are calculated using both Coulomb (velocity) and length gauge (Eqs (16) and (17)). We found [6] that length gauge for R6G calculations is preferable. The level of $10 \%$ accuracy of the convergence of the optical functions could be reached faster then with velocity gauge. This agrees with Rautian's [4] finding. He showed that length gauge is more convenient for confined system, where if using Coulomb gauge, one still needs to sum over wide spectral range. On the other hand we found that for delocalized system (RO-PPV polymer) velocity gauge is more convenient. This approach allows easy corrections to the optical response function. In particular, for several cases in materials optics one needs to incorporate nonnegligible contributions to optical response functions caused by intra-band electron transitions (e.g. for molecular and/or atom surface adsorption, surface/interface contributions etc. [9]) Straightforward use of length gauge in this case causes divergences, which require special treatment [3,9], consequently the Coulomb gauge is preferable.

The Coulomb gauge is very useful by evaluation of Hamiltonian matrix elements in a plane wave basis by using ab initio pseudopotential method [8]. In this case optical response functions (both linear and nonlinear) are evaluated in particularly simple way, which is very important for complex systems [5,9]. However strictly speaking in the optics of materials one can not use momentum operator for the free particle (6). In many-particle system (like molecules or solids) electron experience not only on-site (local) excitations, but also effect of the other particles, which is essentially non-local. This results in the non-locality of momentum operator. Nature of this effect could be understood from the following consideration.

Quite generally the many particles unperturbed Hamiltonian is given by:

$$
H=\frac{\hat{p}_{0}^{2}}{2 m}+V_{l o c}(\vec{r})+\sum\left(\vec{r}, \vec{r}^{\prime}\right)
$$

where operator $\sum\left(\vec{r}, \vec{r}^{\prime}\right)$ represents non-local part of the potential energy of the Hamiltonian.

Momentum (or velocity) operator is given by:

$$
\hat{p}=m \frac{d}{d t} \vec{r}=m \frac{i}{\hbar}[\hat{H}, \vec{r}]=m \frac{i}{\hbar}[(\hat{H} \vec{r})-(\vec{r} \hat{H})]
$$

Taken into account that $\left[V_{l o c}(\vec{r}), \vec{r}\right]=0$ and using following relation for the kinetic part of the Hamiltonian:

$$
\left(\vec{\nabla}^{2} r-r \vec{\nabla}^{2}\right) \psi=\vec{\nabla}(\psi+r \vec{\nabla} \psi)-r \vec{\nabla}^{2} \psi=2 \vec{\nabla} \psi
$$


The equation (2) results in:

$$
\hat{p}=-i \hbar \vec{\nabla}+m \frac{i}{\hbar}\left[\sum\left(\vec{r}, \vec{r}^{\prime}\right), \vec{r}\right]
$$

Last term in (4) represents correction to the momentum operator of the free particle (6) for non-locality of the potential energy (for the particle in molecule or solid). This contribution results in redistribution of the oscillator strengths which substantially improves predicted shape of optical spectra by bringing them closer to experimental data, as it has been demonstrated for inorganic group IV materials in [13]. This should be borne in mind by analysis of the predicted spectral shape.

Equilibrium atomic configuration of organic semi-conducting conjugated polymer RO-PPV $\left(\mathrm{R}=\mathrm{C}_{10} \mathrm{H}_{21}\right)$ is determined by geometry optimization through minimization of the total energy and it is shown in Fig. 1.

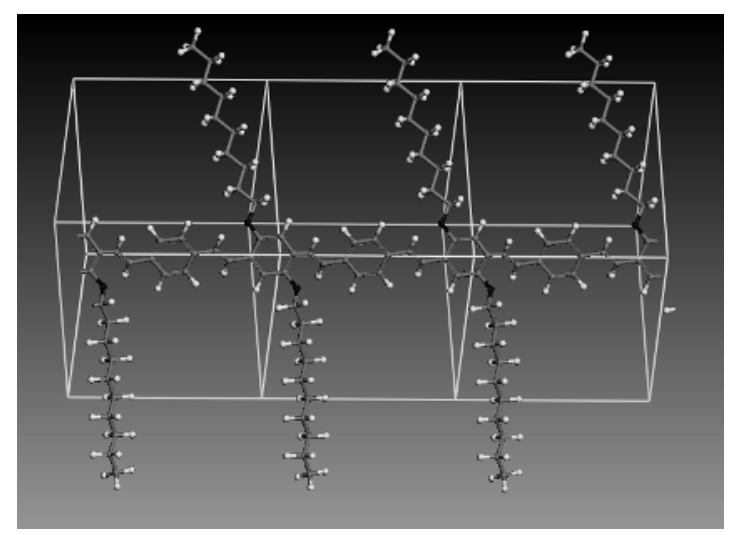

Fig. 1. Equilibrium atomic geometry of RO-PPV conjugated polymer determined through total energy minimization method. By the white line super cell units ordered in one dimensional chain are shown.

Optical absorption spectra of RO-PPV conjugated polymers are calculated using Coulomb gauge from Eq. (16). The self-consistently calculated eigen-functions and eigen-energies for equilibrium geometries are used as inputs. Calculated optical absorption spectrum of PPV single chain conjugated polymer is shown in Fig. 2 . Absolute values of the absorption coefficient are normalized according to the molecular concentration followed from the dimension of the super cell.

Optical absorption of the light polarized along polymer chain $\vec{E} \| \vec{z}$ (see Fig. 2) is more then an order of magnitude stronger than that for perpendicular geometry. Absorption peak around $440 \mathrm{~nm}$ arises from the excitations of delocalized $\pi$-electrons from benzene rings. The basic PPV polymer chain does not show any optical absorption for perpendicular geometry in a visible range [2]. The RO groups slightly change symmetry of the delocalized $\pi$-electrons thus allowing non-zero optical absorption for $\vec{E} \perp \vec{z}$ in a visible range. The quasi-particle correction is applied in the 


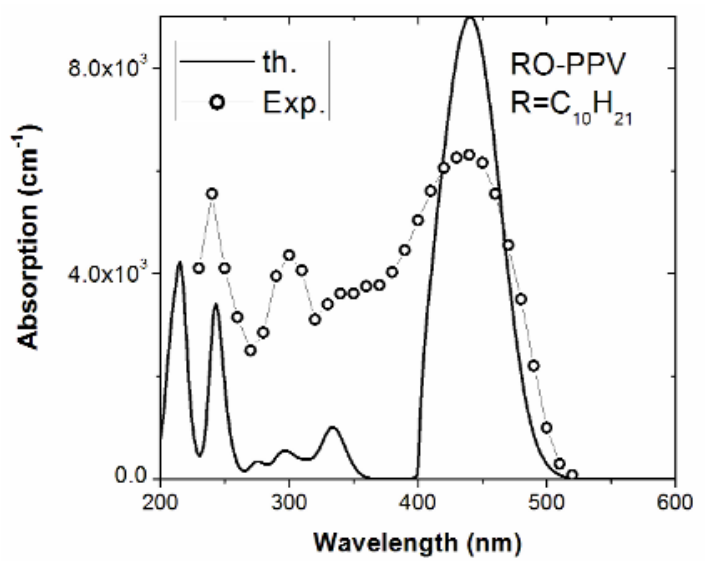

Fig. 2. Calculated optical absorption spectrum of RO-PPV conjugated polymer calculated for parallel ( $\vec{E} \| \vec{z}$ ) geometry. Symbols represent measured optical absorption spectra [14].

form of scissor operator: the predicted anti-bounding electronic states are increased in energy by $0.79 \mathrm{eV}$ to match the $440 \mathrm{~nm}$ measured absorption peak. With this ad hoc QP correction the predicted optical absorption spectrum of RO-PPV correctly represents all main features seen in the measured spectrum (see Fig. 2). Calculated absorption spectrum is close to that observed in similar PPV polymers [4, 15]. However absolute intensities of the absorption peaks deviate strongly from experimental data. Similar situation was reported on simple polymers by using first principle many-body excitonic theory [2]. Better agreement we obtained for R6G dye molecular dimers [6]. The reason for the discrepancy between the shapes of predicted and measured absorption spectra could be the neglect of the momentum operator nonlocality as discussed above. Inclusion of this effect into optical calculation improves agreement with experimental spectral shape for inorganic solids [13].

In [2] full $a b$ initio computations of excitation energies of some simple polymers demonstrated importance of excitonic effects in optics. Such large scale computations within first principle theory (the DFT with QP and many-body exciton corrections [2]) are still challenging for computational physics of complex organic materials. Therefore the first principle pseudopotential method with the $a d$ hoc QP scissor correction for DFT-GGA excitation energies in organic polymers is a reasonable compromise between accuracy and complexity of computations, as demonstrated in this work. Results of the present work as well as that presented in the literature show that overall agreement between predicted and experimental optical spectra in polymers is worse than in solids. One important reason is the reduction of the translational symmetry to only one dimension in polymers. Consequently the increase of the unit cell requires more extensive computational work to achieve the same accuracy level as in three dimensions. Further development of computational methods combining that of physics and chemistry may solve the problem. 


\section{Conclusions}

Method of optical functions calculations of organic molecular complexes based on $a b$ initio pseudopotentials is described. Optical excitation energies are correctly predicted using DFT-GGA method with scissor operator for quasi particle correction. Numerical results obtained for RO-PPV conjugated polymer are in reasonable agreement with experimental data. Effects of gauge invariance and non-locality of optical momentum operator on predicted optical response functions are analyzed.

\section{Acknowledgement}

Author is thankful for discussions with M. Noginov and C. Bonner. This work is supported by NSF grant CREST supplement No. 0520208.

\section{References}

1. Kohn W., Sham, L., J. Phys. Rev., 140 (1965) A1133-A1143

2. Rohlfing M., Luie S. G., Phys. Rev. Lett. 82 (1999) 1959-1962

3. Onida G., Reining L., Rubio A., Rev. Mod. Phys. 74 (2002) 601-633.

4. Barford W., Electronic and Optical Properties of Conjugated Polymers, Oxford University Press, New York (2005)

5. Gavrilenko V. I., Bechstedt F., Phys. Rev. B 55 (1997) 4343-4352

6. Gavrilenko V. I., Noginov M. A., J. Chem. Phys. 124 (2006) 44301-44306

7. Material Studio Modeling 3.2, Accelrys Software Inc. 2004.

8. Fuchs M., Schefler M., Comput. Phys. Commun., 16 (1999) 1-18

9. Gavrilenko V. I., Phys. Status Solidi (a), 188, (2001) 1267-1280

10. Troullier N., Martins J. L., Phys. Rev. B, 43 (1991) 1993-1998

11. Perdew J. P., Burke K., Ernzerhof M., Phys. Re. Lett., 77 (1996) 3865-3875

12. Yu P., Cardona M., Fundamentals of Semiconductors, Springer-Verlag, Berlin Heidelberg New York (2001)

13. Adolph B, Gavrilenko V. I., Tenelsen K., Bechstedt F., Del Sole R., Phys. Rev. B, 53 (1996) 9797-9808.

14. Bonner C. E., Jr., Charter S., Lorts A., Gavrilenko V.I. SPIE Int. Conf. on Optics and Photonics, (San Diego 2006), (submitted).

15. Frolov S., Bao Z., Wohlgenannt M., Vardeny Z.V., Phys. Rev. B 65 (2002) 205209205218

\section{Appendix: Effect of Gauge Invariance on Evaluation of Optical Susceptibility Functions}

Perturbed Hamiltonian of the particle in electro-magnetic field of light is given by:

$$
H=\frac{1}{2 m}\left[\hat{p}+\frac{e}{c} \vec{A}(\vec{r}, t)\right]^{2}+V(\vec{r}),
$$


The momentum operator of free particle is given by:

$$
\vec{p}_{0}=-i \hbar \vec{\nabla}
$$

Using a trial function we have:

$$
\left(\vec{A} \hat{p}_{0}\right)-\left(\hat{p}_{0} \vec{A}\right)=i \hbar \operatorname{div} \vec{A}
$$

Assuming Coulomb gauge ( $\operatorname{div} \vec{A}=0$ ) and bearing in mind that in the light wavelength range up to vacuum ultraviolet the quadratic term on vector-potential in (5) is very small [12], we have:

$$
H=\frac{\hat{p}_{0}{ }^{2}}{2 m}+\frac{e}{m c}\left(\vec{A} \hat{p}_{0}\right)+V(\vec{r})=H_{0}+H_{\mathrm{int}}
$$

with

$$
H_{\mathrm{int}}=\frac{e}{c}\left(\vec{A} \hat{p}_{0}\right)=\frac{e}{m c} A_{o} e^{i(\omega t+\vec{q} \vec{r})}\left(\vec{\tau} \hat{p}_{0}\right)=-i \frac{e}{m \omega} E_{0} e^{i(\omega t+\vec{q} \vec{r})}\left(\vec{\tau} \hat{p}_{0}\right)
$$

where $\tau$ is a unity vector, and for the light wave we have:

$$
\vec{E}=-\frac{1}{c} \frac{\partial \vec{A}}{\partial t}=i \frac{\omega}{c} \vec{A}
$$

Let us consider now the full momentum matrix elements of $p$, determined on the eigen functions of the Hamiltonian (5):

$$
H \psi_{n}=E_{n} \psi_{n}
$$

We have:

$$
\begin{aligned}
p_{\mathrm{ln}} & =m\left\langle l\left|\frac{d \vec{r}}{d t}\right| n\right\rangle=m \frac{i}{\hbar}\left(\sum_{n^{\prime}} H_{\ln ^{\prime}} r_{n^{\prime} l}-\sum_{n^{\prime \prime}} r_{\ln ^{\prime \prime}} H_{n^{\prime \prime} n}\right) \\
& =m \frac{i}{\hbar}\left(E_{l}-E_{n}\right) r_{\ln }=i m \omega_{\ln } r_{\ln }
\end{aligned}
$$

This expression is general. In the length gauge the interaction Hamiltonian is given by [9]:

$$
H_{\mathrm{int}}=e \vec{r} \vec{E}=e E_{0} e^{i(\omega t+\vec{q} \vec{r})}(\vec{\tau} \vec{r})
$$

Based on (9) and (12) the ratio between Coulomb and length gauge interaction Hamiltonians is given by: 


$$
\begin{gathered}
\frac{H_{\mathrm{int}}^{C}}{H_{\mathrm{int}}^{L}}=-i \frac{1}{m \omega} \frac{(\vec{\tau} \hat{p})}{(\vec{\tau} \vec{r})} \\
\frac{\left\langle l\left|H_{\mathrm{int}}^{C}\right| n\right\rangle}{\left\langle l\left|H_{\mathrm{int}}^{L}\right| n\right\rangle}=-i \frac{1}{m \omega} \frac{i m \omega_{\mathrm{ln}} \vec{r}_{\mathrm{ln}}}{\vec{r}_{\mathrm{ln}}}=\frac{\omega_{\mathrm{ln}}}{\omega}
\end{gathered}
$$

Last expression agrees with that given in [4]. It shows that in resonance (if $\omega_{l n}=\omega$ ) both Coulomb and length invariance are equivalent, but out of resonance they are different. Based on time dependent perturbation theory expressions for imaginary part of dielectric permittivity in both gauges are given by $[9,12]$ for Coulomb and length gauges, respectively:

$$
\begin{aligned}
& \varepsilon_{2}(\omega)=\left(\frac{2 \pi e}{m \omega}\right)^{2} \sum_{l, n}\left|p_{\ln }\right|^{2} \delta\left(E_{l}-E_{n}-\hbar \omega\right) \\
& \varepsilon_{2}(\omega)=(2 \pi e)^{2} \sum_{l, n}\left|r_{\ln }\right|^{2} \delta\left(E_{l}-E_{n}-\hbar \omega\right)
\end{aligned}
$$

